\title{
Achievable Diversity and Multiplexing in Multiple Antenna Systems with Quantized Power Control
}

\author{
Ahmad Khoshnevis and Ashutosh Sabharwal \\ Department of Electrical and Computer Engineering \\ Rice University \\ Houston, Texas 77005 \\ Email: \{farbod,ashu\}@ rice.edu
}

\begin{abstract}
We consider a multiple antenna system with finite rate feedback, in which the quantized channel state information at the transmitter is used solely for temporal power control. We show that similar to systems without feedback, the tradeoff between diversity order and multiplexing gain exists. However, unlike the systems with feedback that apply both rate and power control, systems with only power control are unable of achieving non-zero diversity order at the maximum multiplexing gain. The analysis is based on asymptotic behavior of the distribution of order statistics of the eigenvalues of channel matrix, which is a key step in evaluating the diversity order.
\end{abstract}

\section{INTRODUCTION}

It is now well established that channel state information (CSI) leads to significant gains in fading channels. Representative results include improved capacity [1] and large reductions in outages [2]. In fact, the diversity order of a system equipped with perfect CSI at transmitter (CSIT) is exponentially more than that of the diversity order of systems without CSIT [3]. Even with finite amount of feedback information, the diversity order of multiple antenna systems is significantly higher than those of systems without any instantaneous CSIT. ${ }^{1}$

In [4], we showed that much like feedback-free systems [5], there is a tradeoff between diversity order and multiplexing gain in multiple antenna systems with finite amount of feedback information. Given the results of [5] and [6], the existence of tradeoff is not surprising for finite feedback systems, but its specific form is. The most notable is that even with full multiplexing, a non-zero diversity order can be achieved with only one-bit of feedback. In contrast, without feedback the diversity order with full multiplexing cannot be more than zero. Thus, feedback allows the possibility of building full multiplexing MIMO systems without having to completely forego protection due to diversity. The key technique in the proof (see [4] for details) was a combined rate and power control.

In this paper, we continue our analysis of finite feedback multiple antenna systems. Specifically, we show that the rate control mechanism proposed in [4] appears to be the key in obtaining a non-zero diversity order for the case of full multiplexing. Rate control is akin to using different codebooks for different channel conditions. Without rate control, all feedback

\footnotetext{
${ }^{1}$ A $3 \times 3$ system with 1-bit of feedback has a diversity order of 90 , compared to no feedback diversity order of 9 .
}

is used to adapt only the power level of the transmissions. Since the rate of transmission is growing with $\operatorname{SNR}(R \sim$ $r \log S N R$, where $r$ is maximum possible multiplexing gain), time or spatial power control is unable to provide any nonzero diversity gain. Thus, the use of at least two codebooks like in [4] appears to be critical to avoid zero diversity gains.

We consider a multiple antenna system in which channel is represented with finite number of bits to the transmitter through a delay-free and error-free feedback channel. The transmitter uses the quantized information to perform power control (and not rate control). We address the design of channel quantizer and evaluate the diversity order and multiplexing gain of multiple antenna systems with finite rate feedback. For the above system, we quantify the tradeoff between multiplexing gain and diversity order.

The rest of the paper is organized as follows. In Section II along with modeling the system and defining the notations, we will explain the previous and related results. In Section III the main result of the paper is derived analytically. A brief summery of the results and methodology of this work with a glance on possible future directions concludes this paper in Section IV.

\section{PREliminaries}

In a multiple antenna system with $t$ transmit and $r$ receive antennas and block fading channel, the coherence time of the channel is assumed to be much larger than the time required for the transmission of a codeword. The input-output relationship of such a system can be modeled in the matrix notation as

$$
Y=H X+W
$$

where $Y$ is an $r \times 1$ complex vector of output signals, $H \in$ $\mathcal{C}^{r \times t}$ is the channel matrix with independent and identically distributed (iid) entries each with circular complex Gaussian distribution, $X$ is the $t \times 1$ vector of input signals, and $W \in$ $\mathcal{C}^{r \times 1}$ is the vector of additive white complex Gaussian noise with iid entries. Furthermore, it is assumed that the channel matrix $H$ is perfectly known at the receiver, and an error/delay free feedback link exists between the sender and the receiver.

At the receiver, a finite rate channel quanitzer maps the channel matrix $H$ into a finite number, $L$, of subsets. Then the quantized channel is being represented with $B=\log _{2}(L)$ 
bits at the transmitter. Based on the received information about the channel state, transmitter adopts a transmission strategy that optimizes the desired metric. The metrics that we are considering in this paper are multiplexing gain as defined in [5] and given by

$$
r=\lim _{P_{a v} \rightarrow \infty} \frac{R\left(P_{a v}\right)}{\log \left(P_{a v}\right)}
$$

and diversity order defined as

$$
d=\lim _{P_{a v} \rightarrow \infty}-\frac{\log \left[\Pi\left(R\left(P_{a v}\right), P_{a v}\right)\right]}{\log \left(P_{a v}\right)} .
$$

Multiplexing gain $r$ shows how fast the maximum achievable rate $R\left(P_{a v}\right)$ grows with average available power $P_{a v}$. The diversity order $d$ indicates how fast the outage probability $\Pi\left(R\left(P_{a v}\right), P_{a v}\right)$, which is equivalent to the probability of codeword error, decays as the average available power increases.

In this section first we explain the power allocation strategy and the choice of parameter that represents the channel matrix in Section II-A. In Section II-B we find a suboptimum but more tractable channel quantizer which performs close to the optimum one. Through an asymptotic analysis in Section II$\mathrm{C}$ we find the properties of the quantized parameter that will help us in characterizing the outage behavior of the system.

\section{A. Choice of Quantized Parameter}

The optimum combination of the quantizer class (scalar or vector) and the quantized parameter (such as eigenvalues, eigenvectors, space of complex matrices $\mathcal{C}^{r \times t}, \ldots$ ) that optimizes a given performance metric is still an open problem. For design simplicity we consider an scalar quantizer which quantizes one of the eigenvalues of the Wishart matrix $Z$ defined by $Z=H H^{\dagger}$ if $r<t$ and $Z=H^{\dagger} H$ otherwise. Although the selection of an scalar quantizer is arbitrary in here, the choice of parameter requires some justification.

Let $m=\min (t, r)$ and $n=\max (t, r)$. We can represent the channel matrix $H$ by its singular value decomposition as $H=U D^{1 / 2} V^{\dagger}$, where $U \in \mathcal{C}^{r \times m}$ and $V^{\dagger} \in \mathcal{C}^{m \times t}$ are unitary matrices and $D=D^{1 / 2} D^{1 / 2}$ is a diagonal matrix with the eigenvalues of $Z, \lambda_{i}$ for $i \in\{1,2, \ldots, m\}$, on its main diagonal. Since the channel matrix $H$ is known at the receiver, the effect of $U$ can be compensated by multiplying the received signal by $U^{\dagger}$ from left. So we get an equivalent model for the system as

$$
\tilde{Y}=D^{1 / 2} V^{\dagger} X+\tilde{W} .
$$

The modified model of (4) suggests that ideally transmitter needs the information about both $D$ and $V$ matrices. Knowledge of $D$ enables the sender to perform temporal power allocation, and information about $V$ enables transmitter on spatial power allocation or beamforming. As it is shown in [7], the beamforming neither changes the diversity order nor the multiplexing gain of the system. Hence, one might as well adopts a fixed spatial power allocation, such as equally distributing power on all the transmit antennas, and reduce the complexity of the feedback. Let assume that at time $k$ power $P_{k}(H)$, corresponding to channel matrix realization $H$, is being equally divided on the $t$ transmit antennas, and signals on the transmit antennas are iid with Gaussian distribution, then for the covariance of the input signal we have

$$
Q=\mathbb{E}\left\{X X^{\dagger}\right\}=\frac{P_{k}(H)}{t} I_{t},
$$

where $I_{t}$ is the $t \times t$ identity matrix. The instantaneous mutual information expression for the MIMO system with above input distribution is derived in [8] and is given by

$$
\begin{aligned}
I(X ; Y) & =\log \operatorname{det}\left[I_{r}+H Q H^{\dagger}\right] \\
& =\log \operatorname{det}\left[I_{r}+\frac{P_{k}(H)}{t} H H^{\dagger}\right] \\
& \stackrel{a}{=} \log \operatorname{det}\left[I_{r}+\frac{P_{k}(H)}{t} D^{1 / 2}\left(D^{1 / 2}\right)^{\dagger}\right] \\
& =\log \operatorname{det}\left[I_{r}+\frac{P_{k}(H)}{t} D\right] \\
& =\sum_{i=1}^{m} \log \left[1+\frac{P_{k}(H)}{t} \lambda_{i}\right],
\end{aligned}
$$

where $(a)$ is obtained by replacing $H$ with its singular value decomposition.

For a given rate $R\left(P_{a v}\right)$ the outage probability is given by

$$
\Pi\left(R\left(P_{a v}\right), P_{a v}\right)=\operatorname{Pr}\left\{I(X ; Y)<R\left(P_{a v}\right)\right\} .
$$

Replacing Equation(6) into (7) results in

$\Pi\left(R\left(P_{a v}\right), P_{a v}\right)=\operatorname{Pr}\left\{\sum_{i=1}^{m} \log \left[1+\frac{P_{k}(H)}{t} \lambda_{i}\right]<R\left(P_{a v}\right)\right\}$.

Equation(8) shows that when power is equally divided on all the transmit antennas, only the knowledge of the eigenvalues of the Wishart matrix $Z$ is sufficient to prevent outage. On the other hand since we are using a scalar quantizer we focus our attention in the case when only one of the eigenvalues is being quantized. In doing so, we consider the order statistics of eigenvalues, that is $0<\lambda_{1}<\lambda_{2}<\cdots<\lambda_{m}$. Then we evaluate the diversity order and multiplexing gain of a system with finite rate feedback, when $\lambda_{i}, i \in\{1,2, \ldots, m\}$, is being fedback to the transmitter.

\section{B. Quantizer Design}

In the rest of the paper we denote the parameter to be quantized by $\lambda$, the power allocation by $P(\lambda)$, and the boundaries of the quantizer intervals by indexed $\gamma$ 's. We aim to design a quantizer that minimizes the outage probability given in (8) subject to a long term power constraint. The long term power constraint is expressed by

$$
\lim _{T \rightarrow \infty} \frac{1}{T} \sum_{k=1}^{T} P_{k}(\lambda) \leq P_{a v}
$$

Assuming that $\lambda$ is a first order ergodic process, then the time average on the LHS of (9) is equal to the ensemble average. Therefore (9) can be rewritten as

$$
\mathbb{E}\{P(\lambda)\} \leq P_{a v}
$$


where the expectation is taken with respect to the distribution of $\lambda$.

In [6] we showed that regardless of the distribution, $f(\lambda)$, of the quantized parameter $\lambda$, dividing the power equally ${ }^{2}$ over the quantization bins is asymptotically (in the feedback rate) optimum, and the outage performance of this suboptimum channel quantizer matches the outage performance of the system with optimum quantizer. The power allocated at all the quantization bins, except the first bin, guarantees outage free communication. The power allocated to the first bin, $P(0)$ can not satisfy all channel conditions in the first bin, $\left[0, \gamma_{1}\right)$. Thus there exists ${ }^{3}$ a $\gamma_{0} \in\left[0, \gamma_{1}\right)$ such that $I(X ; Y)<R\left(P_{a v}\right)$ for $\lambda<\gamma_{0}$. Therefore, the probability of outage is fully characterized by $\gamma_{0}$ as

$$
\Pi\left(R\left(P_{a v}, P_{a v}\right)\right)=\operatorname{Pr}\left(\lambda<\gamma_{0}\right)
$$

The equal total power division explained in this section, provides a systematic algorithm for design of channel quantizer at the receiver, which is asymptotically optimum, and is simple to analyze. We will show in Section III how a simple recursive algorithm can be use to find the diversity order of the system for a given channel parameter $\lambda$.

Since equal spatial power allocation (divide the total power equally on all the transmit antennas) does not require information about the matrix of eigenvectors, $V$, then one can further simplify the system model given in (1) to

$$
\tilde{Y}=D^{1 / 2} \tilde{X}+\tilde{W} \text {. }
$$

Furthermore, there exists a space-time code that achieves the mutual expression in (6). The significance of (12) is that it enables us to view the channel as $m$ parallel channels corresponding to $m$ eigenvalues of the Wishart matrix $Z$. Therefore, if by power allocation one can guarantee a reliable communication on the channel corresponding to the $i^{\text {th }}$ largest eigenvalue, then reliable communication is also available on all channels with larger eigenvalues. Thus, in order to achieve a multiplexing gain of $r$, it is sufficient to have a multiplexing gain of one on the channel corresponding to the $r^{t h}$ largest eigenvalue.

From the above observation it follows that in order to fully characterize the diversity order and multiplexing gain, we only need to determine the diversity order when the $r^{\text {th }}$ largest eigenvalue is chosen to be quantized.

\section{Asymptotic Properties of CDF of Eigenvalues}

The results of this section are proved in [4] and presented here for the sake of completeness. Throughout this paper we assume the order statistics of the eigenvalues of the Wishart matrix $Z$, defined in Section II-A. The joint distribution of the

\footnotetext{
${ }^{2}$ By dividing power equally we mean that if there are $L$ quantization bins, and total available power is $P_{a v}$, then the power allocated to the $i^{\text {th }}$ bin is given by: $P(i-1) \operatorname{Pr}\left\{\gamma_{i-1} \leq \lambda \leq \gamma_{i}\right\}=\frac{P_{a v}}{L}$, for $i=1,2, \ldots, L$.

${ }^{3}$ With channel inversion power allocation we have $\gamma_{0}=\frac{k}{P(0)}$, where $k=\left(2^{R\left(P_{a v}\right)}-1\right) / \sigma^{2}$, and $\sigma^{2}$ is the variance of the additive noise.
}

ordered eigenvalues, $0<\lambda_{1}<\lambda_{2}<\cdots<\lambda_{m}$, is given in [9] by

$$
f\left(\lambda_{m}, \lambda_{m-1}, \ldots, \lambda_{1}\right)=e^{-}{ }_{i=1}^{m} \lambda_{i} \prod_{i=1}^{m} \lambda_{i}^{n-m} \prod_{i<j}\left(\lambda_{i}-\lambda_{j}\right)^{2} .
$$

Let $\lambda_{i}$ be the $i^{\text {th }}$ smallest eigenvalue of $Z$, then the marginal distribution of $\lambda_{i}$ can be obtained by integrating (13) over all $\lambda_{j}$ 's, $j \neq i$. That is

$$
\begin{aligned}
& f\left(\lambda_{i}\right)=\int_{0}^{\infty} \int_{0}^{\lambda_{m}} \cdots \int_{0}^{\lambda_{i+2}} \int_{0}^{\lambda_{i}} \cdots \int_{0}^{\lambda_{2}} \\
& f\left(\lambda_{m}, \ldots, \lambda_{1}\right) d \lambda_{1} \ldots d \lambda_{i-1} d \lambda_{i+1} \ldots d \lambda_{m} .
\end{aligned}
$$

Define $F_{\lambda_{i}}(x)$ to be cumulative density function of $\lambda_{i}$ on the interval $[0, x]$ and be given by

$$
F_{\lambda_{i}}(x)=\int_{0}^{x} f\left(\lambda_{i}\right) d \lambda_{i} .
$$

Although the marginal distribution ${ }^{4}$ of $\lambda_{i}$, for all $i \in 2, \ldots, m$, is not known, the following lemma is a key result in derivation of the diversity order. Note that for simplicity in integration, the analysis so far has assumed an ascending order for eigenvalues. From now on, however, we assume a descending order for the eigenvalues in which $\lambda_{1}$ represents the largest eigenvalue and $\lambda_{i}$ the $i^{\text {th }}$ largest.

Lemma 2.1: The cumulative density function of the $i^{t h}$ largest eigenvalue of a Wishart matrix, $F_{\lambda_{i}}(x)$, admits Taylor series expansion of

$F_{\lambda_{i}}(x)=\beta x^{(n-i+1)(m-i+1)}+o\left(x^{k}\right), \quad k>(n-i+1)(m-i+1)$.

around origin $(x=0)$, for some constant $\beta$.

An immediate consequence of the Lemma 2.1 is on the diversity order derived from the expression for outage and is given in the next corollary.

Corollary 2.2: Consider a system with finite rate feedback in which the $i^{\text {th }}$ largest eigenvalue, $\lambda_{i}$, is quantized at the receiver and fedback to the transmitter. Then as the average available power increases unboundedly, the probability of outage is given by

$$
\begin{aligned}
\Pi\left(R, P_{a v}\right) & =\operatorname{Pr}\left\{\lambda<\gamma_{0}\right\} \\
& =F_{\lambda_{i}}\left(\gamma_{0}\right) \\
& \approx \gamma_{0}^{(n-i+1)(m-i+1)}
\end{aligned}
$$

In Section III we will show that how $\gamma_{0}$ depends on the $P_{a v}$. Doing so, enables us to completely characterize the diversity order for a given multiplexing gain.

\section{MAIN RESULT}

In this section we use the suboptimum quantizer developed in [6] to evaluate the diversity order for a given multiplexing gain. As mentioned in Section II-B, if a multiplexing gain of

\footnotetext{
${ }^{4}$ The distribution of the $\lambda_{1}$ corresponding to the smallest eigenvalue is derived in [9]
} 
1 is achievable on the channel corresponding to the $i^{t h}$ largest eigenvalue, then a multiplexing gain of 1 is also achievable on channels $1, \ldots, i-1$. Thus a total multiplexing gain of $i$ is achievable by controlling the multiplexing gain of the $i^{t h}$ channel. The outage probability, when the $i^{t h}$ largest eigenvalue is quantized, is given in (17). In order to find the multiplexing gain, we need to find $\gamma_{0}$ as a function of $P_{a v}$. Lemma 3.2 establishes such relation.

Before stating the lemma we should define and fix the frame work of the system that we are considering through out the paper.

Definition 3.1: Let system parameters $t, r, m, n$, feedback parameters $L, B$, and Wishart matrix $Z$, all defined in previous sections. Furthermore, consider the general feedback case in which the $i^{\text {th }}$ largest eigenvalue of $Z$ is the quantization parameter, and a multiplexing gain of $\alpha \in[0,1]$ is achieved on the corresponding channel. That is the rate at the channel of the $i^{t h}$ largest eigenvalue is asymptotically (in $P_{a v}$ ) is given by $R\left(P_{a v}\right)=\alpha \log \left(P_{a v}\right)$.

Lemma 3.2: Consider a system with parameters given by definition 3.1. Define $G(m, n, i, L)$ by

$$
G(m, n, i, L)=\sum_{l=0}^{L-1}[(n-i+1)(m-i+1)]^{l} .
$$

Then $\gamma_{0}(i)$ ( $\gamma_{0}$ corresponding to the quantizer of the $i^{t h}$ largest eigenvalue) is

$$
\gamma_{0}(i) \approx \frac{c_{i}}{P_{a v}^{(1-\alpha) G(m, n, i, L)}}
$$

for large values of $P_{a v}$, and some constant $c_{i}$.

Proof: Analyzing the suboptimum quantizer, as described in Section II-B, with equal total power at each quantization bin, and beginning from the last quantization bin we have

$$
\begin{aligned}
\frac{P_{a v}}{L} & =P(L-1) \operatorname{Pr}\left\{\gamma_{L}<\lambda_{i}<\infty\right\} \\
& \stackrel{a}{=} \frac{2^{R\left(P_{a v}\right)}-1}{\gamma_{L}}\left[F_{\lambda_{i}}(\infty)-F_{\lambda_{i}}\left(\gamma_{L}\right)\right] \\
& \stackrel{b}{=} \frac{P_{a v}^{\alpha}}{\gamma_{L}}\left[1-F_{\lambda_{i}}\left(\gamma_{L}\right)\right]
\end{aligned}
$$

where (a) is obtained by replacing power allocated to the $L^{\text {th }}$ bin $^{5}, P(L-1)$, by its channel inversion equivalent and (b) is obtained by having $R\left(P_{a v}\right)=\alpha \log \left(P_{a v}\right)$. Solving (20) we have

$$
\gamma_{L} \approx \frac{c_{1}}{P_{a v}^{1-\alpha}}
$$

Note that having $\gamma_{L}$ from (21), the expression of power allocated at the $L-1^{s t}$ bin given by

$$
\frac{P_{a v}}{L}=P(L-2) \operatorname{Pr}\left\{\gamma_{L-1}<\lambda_{i}<\gamma_{L}\right\},
$$

is only a function of $\gamma_{L-1}$. Solving (22) and repeating the procedure for all the bins sequentially toward the first bin yields the statement of the lemma.

\footnotetext{
${ }^{5}$ Note that quantization bins and corresponding power levels are denoted by $\left[0, \gamma_{1}\right),\left[\gamma_{1}, \gamma_{2}\right), \ldots,\left[\gamma_{L}, \infty\right)$ and $P(0), P(1), \ldots, P(L-1)$ respectively.
}

It is enough to replace (22) into (17) to obtain the relation between probability of outage, average power, and the multiplexing gain. The resulting probability of outage is an upper bound on actual outage probability, which can be easily tightened by following observation.

Consider that a multiplexing gain $j$ with $j \in\{1,2, \ldots, i-$ $1\}$ is desired, when the maximum achievable multiplexing is $i$. Then the outage probability in (17), is the probability of outage of the channel corresponding to $\lambda_{i}$. Whereas we only need that the first $j$ channel, corresponding to $\lambda_{m}, \ldots, \lambda_{m-j+1}$ not being in outage. Hence the probability of outage is given by

$$
\begin{aligned}
\Pi\left(R\left(P_{a v}\right), P_{a v}\right) & =\operatorname{Pr}\left\{\lambda_{j}<\gamma_{0}(i)\right\} \\
& =\gamma_{0}(i)^{(n-j+1)(m-j+1)} \\
& =\left(\frac{\beta}{P_{a v}^{(1-\alpha) G(m, n, i, L)}}\right)^{(n-j+1)(m-j+1)}
\end{aligned}
$$

From Equation(23) the relationship between multiplexing gain and the diversity order can be extracted. Moreover, for a given maximum multiplexing gain of $i$, one can find the diversity orders for intermediate integer multiplexing gains of $j<i$. Note that when the system provides full multiplexing gain $(\alpha=1)$ the diversity order is zero, and for multiplexing gain of $j<\eta<j+1, j \in \mathbb{N}$ (which corresponds to $\alpha=\eta / i$ ) the diversity order linearly increases and there is a discontinuity at each integer multiplexing gain. The above deductions from (23) are illustrated in Figure 1. The overall achievable diversity-multiplexing curve is the outer envelop of all the curves as depicted in Figure 1 with solid line.

The diversity order and multiplexing gain of a MIMO system with finite rate feedback developed in the above can be summarized in the following theorem.

Theorem 3.3: Consider a MIMO system with parameters as defined in definition 3.1. For a multiplexing gain $r$, when a scalar quantizer is used at the receiver and a single codebook is used at the transmitter, the diversity order is given by

$$
d=\max _{i \in\{j, \ldots, m\}}\{(1-\alpha)(n-j+1)(m-j+1) G(m, n, i, L)\},
$$

where $j=\lceil r\rceil$ and $\alpha=r / i$.

Proof: Proof is straight forward by the results of Lemma 3.2, the diversity order extracted from Equation(23), and finding the maximum over all possible diversity orders for a given multiplexing gain when different eigenvalue is being quantized.

Figure 1 depicts the diversity-multiplexing curve for a system with 1 bit of feedback, $m=3$, and $n=4$. Also for the sake of comparison the diversity-multiplexing curve of system without feedback, as given in [5], is plotted. There are three sets of curves in Figure 1. The solid curve, dashed curves with circle markings, and dashed with triangle. The dashed curve with triangle markings at the bottom is the diversitymultiplexing curve of a system without feedback. The solid envelope is the maximum diversity-multiplexing achievable by a system with finite rate feedback. The discontinuities at the integer multiplexing gains are due to the switching the 


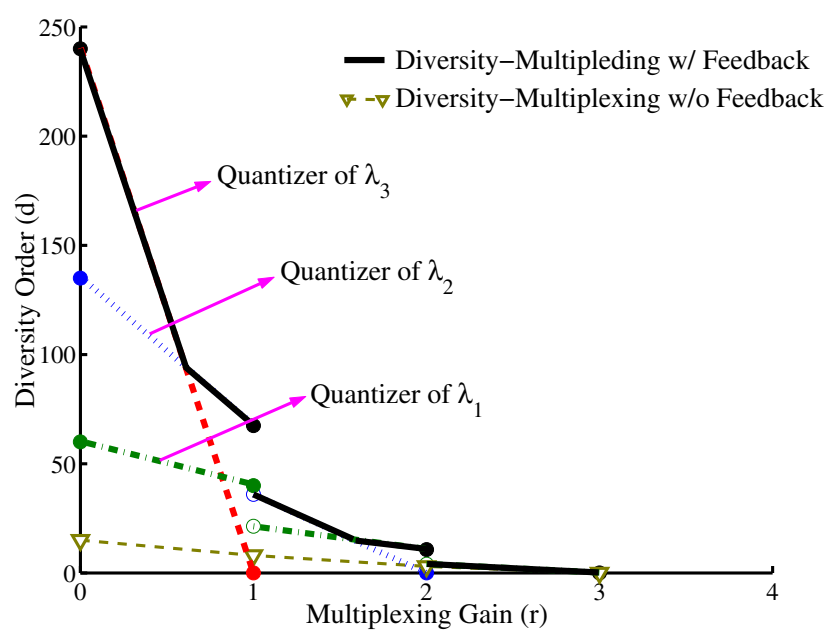

Fig. 1. Diversity and Multiplexing curve with 1 bit of feedback and without feedback as in [5], for system with $m=3, n=5$.

channel quantizer to a smaller eigenvalue. The set of dashed lines with circle markings can be divided into three curves. Since $m=3$, there are three eigenvalues that can be used to design the channel quantizer. The curve with highest slope that crosses the multiplexing axis at $r=1$ corresponds to the quantization of $\lambda_{3}$, which is the largest eigenvalue. The dotted curve with smaller slope that crosses the multiplexing axis at $r=2$ and has a discontinuity at $r=1$ corresponds to the quantization of $\lambda_{2}$, the second largest eigenvalue. When a multiplexing gain larger than 1 is desired, the outage is determined by the realization of $\lambda_{2}$, whereas for multiplexing gains smaller than one it suffices to receive data reliably on the channel corresponding to $\lambda_{3}$. Therefore, the outage behavior is determined by the realization of $\lambda_{3}$. An analytical description of the above is given in (23). Finally the dashed and dotted curve with the smallest slope (still with circle marking) that crosses the multiplexing axis at $r=3$ corresponds to the quantization of $\lambda_{1}$, which is the smallest eigenvalue. Similarly there are discontinuities at intermediate integer multiplexing gains of $r=1,2$.

Figure 2 depicts the diversity-multiplexing curves for a system using a single codebook (solid line) and a system with two codebooks [4] (dashed line). As illustrated in Figure 2, systems with single codebook have a higher diversity order except at high multiplexing gains. The diversity order of systems with single codebook is zero as the system exploits all the possible parallel channels, whereas in systems with two codebooks, a non-zero diversity order is achieved while benefiting from the highest available multiplexing gain.

As the number of bits in feedback increases, the envelope curve stretches vertically. Hence, one can expect that as the number of feedback bits approaches infinity, i.e., perfect channel state information at the transmitter, the diversity gain approaches infinity for any arbitrary multiplexing gain.

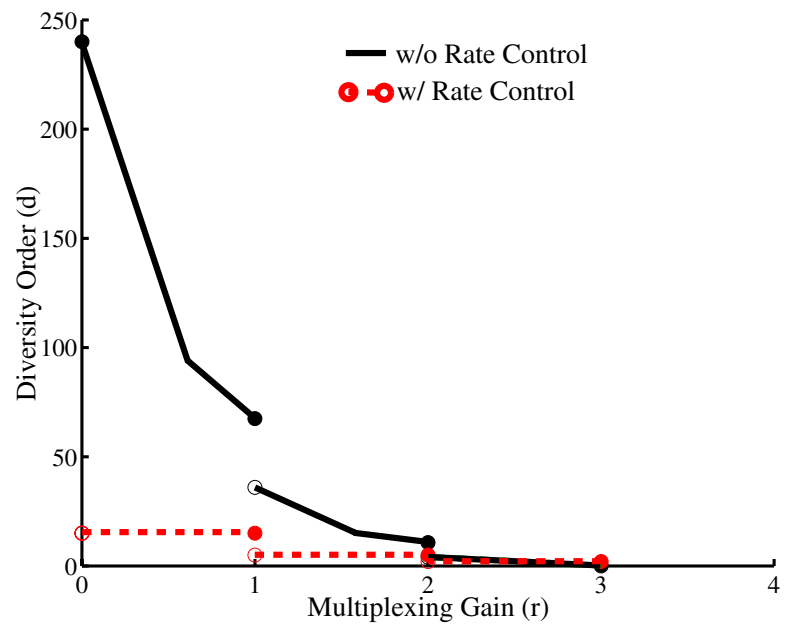

Fig. 2. Diversity and Multiplexing curves with $m=3, n=5$, and 1 bit of feedback, for systems with and without rate control

\section{CONCLUSIONS}

We derived the diversity order and multiplexing gain of a MIMO system with finite rate feedback. The transmitter utilizes the channel state information for power control. Analysis results show that such a system has zero diversity order at the maximum multiplexing gain. This is in contrast to the diversity-multiplexing tradeoff of MIMO systems in which the finite rate feedback at the transmitter is used for both power and rate control. In summary, two significant factors in design of a wireless communication system can be identified as long term temporal power control to benefit from large diversity order, and adaptive rate-power control to achieve non-zero diversity order at maximum multiplexing gain.

\section{REFERENCES}

[1] S. K. Jayaweera and H. V. Poor, "Capacity of multiple-antenna systems with both receiver and transmitter channel state information," IEEE Trans. on Inform. Theory, vol. 49, no. 10, pp. 2697-2709, Oct. 2003.

[2] G. Caire, G. Taricco, and E. Biglieri, "Optimum power control over fading channels," IEEE Transactions on Information Theory, vol. 45, no. 5, pp. 1468-1489, July 1999.

[3] K. Premkumar, A. Rangarajan, and V. Sharma, "Exponential diversity achieving spatio-temporal power allocation scheme for mimo fading channels,' in IEEE Int. Symp. Inform. Theory, ISIT'04, Chicago, IL, June 27-July 1 2004, pp. 803-807.

[4] A. Khoshnevis and A. Sabharwal, "On diversity and multiplexing gain of multiple antenna systems with transmitter channel information," in Forty-Second Annual Allerton Conf. on Comm., Control, and Comp., Monticello, Il, Sep. 29-Oct. 12004.

[5] L. Zheng and D. N. C. Tse, "Diversity and multiplexing: A fundamental tradeoff in multiple-antenna channels," IEEE Trans. on Inform. Theory, vol. 49, no. 5, pp. 1073-1096, May 2003.

[6] A. Khoshnevis and A. Sabharwal, "Performance of quantized power control in multiple antenna systems," in IEEE Int. Conf. on Communications, ICC'04, vol. 2, Paris, France, June 20-24 2004, pp. 803-807.

[7] K. K. Mukkavilli, A. Sabharwal, E. Erkip, and B. Aazhang, "On beamforming with finite rate feedback in multiple antenna systems," IEEE Trans. Inform. Theory, vol. 49, no. 10, pp. 2562-2579, Oct. 2003.

[8] I. E. Telatar, "Capacity of multi-antenna gaussian channels," AT\&T Bell Laboratories, Tech. Rep., June 1995.

[9] A. Edelman, "Eigenvalues and condition numbers of random matrices," Ph.D. dissertation, Massachusetts Institute of Technology, MIT, May 1989. 